\title{
¿El hacer médico necesita del pensar filosófico?
}

DOI: https://doi.org/10.33262/ap.v3i4.110

\author{
(c) (i) (9) (2) \\ Does making medical need of thinking philosophical? \\ Caridad J. Fernández Valderrama. ${ }^{1}$, Bárbara Rodríguez Álvarez. ${ }^{2}$, Zulay \\ Fernández Garrido. ${ }^{3}$ \& Efrain Velastegui López. ${ }^{4}$
}

\begin{abstract}
Today, as in no previous era, the transcendence that technological scientific development has acquired in the life of society, in all areas of its existence, is incomparable, this is an impossible fact to ignore, and it necessarily leads to a reflection on the implications that poses such a problem for life on the planet. A world that is transformed with unusual rapidity as a result of human actions should necessarily be embodied in respect for the essential values and rights of the individual and of everything that lives. It is not possible to ignore the social responsibility of the scientist before his investigative results, it is not possible today, to do science detached from a humanist conception centered on respect for all that is alive and for the value of human dignity; It is not possible to ignore the philosophical reflection that involves the treatment that implies the consequences of technological scientific development whatever the spheres of knowledge that are analyzed. However, even when it seems that such an understanding of the subject is accepted by a large number of scholars, it is significant to observe how disdainful attitudes are still found towards the need for dialogue between philosophical thinking and scientific endeavor, even in directly related areas of knowledge. With the life of man as are the medical sciences. This situation has motivated the development of this reflection, with
\end{abstract}

\footnotetext{
1 Doctora en Ciencias Económicas. Universidad de La Habana, Facultad de Turismo, Departamento Turismo , Facultad de Ciencias Médicas “General Calixto García”, Departamento Filosofía e Historia, email: valderrama@ftur.uh.cu, cafeval3000@gmail.com, ORCID. ID https://orcid.org/0000-0002-79864578

2 Doctora en Ciencias Filosófica. Universidad de La Habana, Facultad de Turismo, Departamento Turismo, email: rodriguezbarbara785@gmail.com, ORCID. ID https://orcid.org/0000-0002-4115-1927

${ }^{3}$ Centro de Ingeniería Genética y Biotecnología. Planta de Producción, e.m: zulay.fernandez@cigb.edu.cu ORCID. ID https://orcid.org/0000-000-6584-9708.

4 Universidad Técnica de Babahoyo, Facultad de Ciencias de la Educación, Los Ríos, Babahoyo, evelasteguil@utb.edu.ec, ORCID https://orcid.org/0000-0002-7353-5853
} 
the sole purpose of deepening once more the need for the relationship between Philosophy and Medicine.

Keywords: Philosophy, Medicine, Science, Health.

\section{Resumen}

Hoy como en ninguna época anterior resulta incomparable la trascendencia que ha adquirido el desarrollo científico tecnológico en la vida de la sociedad, en todos los ámbitos de su existencia, ese es un hecho imposible de ignorar, y lleva obligatoriamente a una reflexión sobre las implicaciones que plantea tal problemática, para la vida en el planeta. Un mundo que se transforma con una rapidez inusitada como resultado del actuar humano forzosamente debería concretarse en el respeto hacia los valores y derechos esenciales del individuo y de todo lo vivo. No es posible soslayar la responsabilidad social del científico ante sus resultados investigativos, no es posible hoy, hacer ciencia desvinculada de una concepción humanista centrada en el respeto por todo lo vivo y por el valor de la dignidad humana; no es posible desentenderse de la reflexión filosófica que conlleva el tratamiento que implica las consecuencias del desarrollo científico tecnológico cualesquiera sean las esferas del saber que se analice. No obstante, aun cuando pareciera que tal comprensión del tema es admitida por una gran cantidad de estudiosos, resulta significativo observar cómo se encuentran todavía actitudes desdeñosas hacia la necesidad del diálogo entre el pensar filosófico y el quehacer científico, incluso en áreas del conocimiento directamente vinculadas con la vida del hombre como son las ciencias médicas. Esta situación ha motivado el desarrollo de estas líneas con el objetivo de reflexionar sobre la relación entre la Filosofía y la Medicina.

Palabras Claves: Filosofía, Medicina, Ciencia, Salud

\section{Introducción}

Hoy como en ninguna época anterior resulta incomparable la trascendencia que ha adquirido el desarrollo científico tecnológico en la vida de la sociedad, en todos los ámbitos de su existencia, ese es un hecho imposible de ignorar, y lleva obligatoriamente a una reflexión sobre las implicaciones que plantea tal problemática, para la vida en el planeta. Un mundo que se transforma con una rapidez inusitada como resultado del actuar humano forzosamente debería concretarse en el respeto hacia los valores y derechos esenciales del individuo y de todo lo vivo.

No es posible soslayar la responsabilidad social del científico ante sus resultados investigativos, no es posible hoy, hacer ciencia desvinculada de una concepción humanista centrada en el respeto por todo lo vivo y por el valor de la dignidad humana; no es posible desentenderse de la reflexión filosófica que conlleva el tratamiento que implica las consecuencias del desarrollo científico tecnológico cualesquiera sean las esferas del saber que se analice. 
No obstante, aun cuando pareciera que tal comprensión del tema es admitida por una gran cantidad de estudiosos, resulta significativo observar cómo se encuentran todavía actitudes desdeñosas hacia la necesidad del diálogo entre el pensar filosófico y el quehacer científico, incluso en áreas del conocimiento directamente vinculadas con la vida del hombre como son las ciencias médicas.

Esta situación ha motivado el desarrollo de estas líneas con el objetivo de reflexionar sobre la relación entre la Filosofía y la Medicina.

\section{Metodología}

La ruta metodológica de la investigación estuvo presidida por la concepción dialéctico materialista de la realidad, además se emplearon técnicas y métodos como el análisissíntesis, inducción-deducción, lo histórico-lógico para la revisión de las fuentes bibliográficas, se utilizaron fuentes primarias y secundarias de información.

\section{Resultados}

Los argumentos presentados constituyen una propuesta de actualización y profundización de los programas de posgrado de la asignatura Filosofía, Ciencia y Salud para los médicos residentes de la Facultad de Ciencias Médicas General Calixto García, de la Universidad de La Habana.

\section{La relación filosofía-medicina}

Entre los posgrados destinados a completar la preparación de los residentes de medicina en las universidades cubanas, se encuentra el curso de Filosofía, Ciencia y Salud, lamentablemente la mayor parte de los cursistas solo asisten porque es parte del currículo obligatorio a cumplimentar, puesto que no alcanzan a distinguir qué les puede aportar este tipo de saber a su profesión, mucho menos la importancia que tiene en un mundo cada vez más dominado por la robótica, la cibernética, la nanotecnología, la inteligencia artificial, la realidad virtual, la biotecnología, por solo mencionar algunas, incluso algunos de los alumnos llegan a expresar que es una pérdida de tiempo y se plantean una medicina divorciada de la reflexión filosófica, es decir la ciencia médica apartada de la duda, de un profundo razonamiento sobre las implicaciones de su quehacer en el convulso mundo en el que se desenvuelve hoy.

El surgimiento y desarrollo de la medicina no puede comprenderse desvinculado de su estrecha e indisoluble sujeción con el desarrollo de la producción material, cultural, científica, en general, así como por la poderosa influencia que ha tenido la pugna entre las vertientes ideológicas esenciales en cada época.

Desde que comienza a gestarse el pensamiento teórico se establece un apretado vínculo, entre lo que posteriormente serían denominadas las ciencias humanísticas y las ciencias de la salud, ya que su desarrollo estará determinado por las condiciones histórico sociales en que surgieron y van progresando; pero aún hoy, en los escenarios donde acuden especialistas de las ciencias naturales, o ingenieros, o médicos, un número no 
despreciable, olvidan o subestiman esta relación y cuando se declara que necesitan de la filosofía, o de las ciencias sociales y humanísticas, rechazan fuertemente esta aseveración, pues choca con la percepción cotidiana, casi unánimemente compartida por estudiantes, profesores y especialistas que aceptan una división del trabajo científico que aísla a las ciencias entre sí, y tratan de desentenderse más aún de las sociales.

Esta separación se encuentra convenientemente arraigada en el orden institucional vigente, se estudian carreras y posgrados separados, inclusive en centros universitarios diferentes, lo cual no es más que el reflejo de la separación cognitiva que se generó entre las ciencias naturales y las humanísticas; situación que echó raíces con la modernidad y sin lugar a dudas, ha conllevado a un empobrecimiento más y más profundo de los diversos campos del saber; no obstante, ya es tiempo de buscar las articulaciones, las interrelaciones, de reconectar las ciencias entre sí, ya no es sostenible la falsa idea de que el conocimiento alcanzado es exacto, fiel reflejo de los fenómenos y procesos, cuando lo cierto es, que en ese proceso de abstracción científica mediante el cual intentamos conocer la realidad, se escapan múltiples aristas, relaciones, nexos, que solo es posible captar en el movimiento, la interconexión, los intervínculos, en la dinámica interactuante, en la organicidad de los diversos sistemas.

Luego en pleno siglo XXI, ¿es posible que aún se sustenten posiciones que tienden a la separación del nexo medicina-filosofía? ¿es menester seguir desconociendo esta íntima relación?, ¿reconocer este vínculo significa que el médico abandone su profesión para dedicarse a la disquisición filosófica?

A estas inquietantes interrogantes se le han adjudicado una larga lista de respuestas, entre las cuales es destacable la brindada por J. M. Taborda Alzate y F. L. Ochoa Jaramillo quienes se plantean: ... ¿qué implica la actitud filosófica en la vida del médico? Ante todo, entrañaría asumir responsablemente un sólido proceso de formación filantrópica y filotécnica.

El médico está, por naturaleza de su oficio, expuesto siempre a relacionarse con la enfermedad, la vejez y la muerte. Justamente por eso, habría de nutrirse de las manifestaciones más profundamente humanas: la música, la literatura, la filosofía, el arte, la mística. Si el médico reduce su quehacer a una mera labor técnica (filotecnia), no sólo reduce la mirada sobre su paciente, sino que va limitando el sentido de lo que él mismo es (su identidad en tanto ser humano, social y cultural).

En concordancia con el ideal hipocrático, el médico, se tendría que hacer suya la clásica sentencia de Terencio: "Homo sum, humani nihil a me alienum puto" (Soy humano, y nada de lo humano me es ajeno). Así pues, al médico no debería serle ajeno lo político, lo económico, lo estético, lo ético, lo antropológico, lo sociológico, ni tampoco lo filosófico. Y yendo más allá: no solamente lo humano habría de ser considerado por el médico, también lo aparentemente no humano habría de ser objeto de su asombro: la zoología, la botánica, la cosmología, la ecología, etcétera; todas ellas no tendrían por qué ser ajenas al interés del médico en tanto filántropo. (Taborda Alzate Jhon M., 2008) 
Indudablemente, no se intenta un mágico acto de conversión en el que se transforme el médico en filósofo, tampoco se ha de pensar que el filósofo sea el indicado para introducirse en las ciencias médicas, tan sólo se pretende llamar la atención sobre la necesidad de un profundo examen sobre el hombre y su lugar en el universo, y en especial sobre el hacer médico y su relación con el pensar filosófico. De modo tal, que ambos dialoguen y dicho dialogo tribute a la comprensión de la complejidad del mundo en cual están insertos.

\section{Sobre filósofos-médicos o médicos-filósofos.}

Es sabido que la relación entre ambas esferas del saber ha sido compleja, sin embargo, al hurgar en la historia pasada de ambas, se encuentran múltiples ejemplos de médicos en los que ha estado presente una fuerte preocupación filosófica. Y es que sería impensable un médico, cuyos conocimientos sobre el cuerpo humano, su relación con el medio y su influencia en la salud, no se sustenten en una determina concepción del mundo de la cual se deriva la condición ética asumida.

Este íntimo vínculo entre el hombre y el medio circundante, estudiado desde la esfera de la salud, aunque de modo espontáneo e ingenuo estuvo presente en el conocimiento médico del período de la Antigüedad; sobre ello se han encontrado numerosas evidencias, en las investigaciones realizadas de muchos pueblos como por ejemplo en la India, en Egipto, en China y Grecia.

Uno de los primeros pensadores cuyo quehacer en la medicina ha trascendido a través de la historia humana fue Hipócrates, quien a partir de la concepción dialéctica predominante en su época, consideraba a la enfermedad como un proceso general de todo el organismo, por lo que este último debía concebirse en su unidad e integridad, buscaba las causas materiales de las enfermedades y su relación con el medio ambiente, fue capaz de separar la práctica médica de las especulaciones filosóficas y la magia.

Por su parte el pensamiento de Galeno ejerció una profunda influencia en la medicina practicada en el Imperio Bizantino, que se extendió con posterioridad a Oriente Medio para acabar llegando a la Europa medieval, donde vivió hasta entrado el siglo XVII.

Autores como M. Ruiza, T. Fernández, E. Tamaro, entre otros, señalaron que influido por las doctrinas de Hipócrates, Galeno sostuvo como tesis, que la salud del individuo se basa en el equilibrio entre la sangre y una serie de humores conocidos como bilis amarilla, bilis negra y flema. Fue pionero en la observación científica de los fenómenos fisiológicos, y practicó numerosas disecciones, que le permitieron identificar siete pares de nervios craneales, describir las válvulas del corazón e incluso establecer las diferencias estructurales entre venas y arterias. Galeno logró demostrar asimismo que las arterias no transportaban aire, como entonces se creía, sino sangre, (Ruiza, Fernández, \& Tamaro, 2004).

A la postre, Galeno recopiló los conocimientos médicos de su tiempo, en especial la fisiología, la patología farmacología, terapéutica, higiene, obstetricia, entre otras. En 
filosofía bebió de la vertiente idealista de Platón específicamente su teoría sobre el pneuma y de Aristóteles tomó la doctrina de las causas finales. ... "Sus concepciones y teorías contienen muchas especulaciones y poca correspondencia con la realidad" (Campohermoso Rodríguez O, 2017), aun así, ejerció una notable influencia en la medicina posteriormente.

En el período feudal destacaron, Avicena, Averroes y Maimónides, entre otros, quienes conciben a la filosofía como una vía necesaria para la comprensión de muchas problemáticas para las cuales la medicina no había encontrado aún respuesta.

Avicena, famoso sabio y eminente médico de la Edad Media, fue uno de los pensadores más importantes de la denominada filosofía islámica y de la medicina universal, en ellas recreó su capacidad de concentración y su fuerza intelectual, estudió, comentó y revolucionó las obras médicas de antecesores griegos como Hipócrates, Claudio Galeno, Dioscórides, el naturalista y sabio Aristóteles, lo que le proporciona reconocimiento por muchas centurias, como el médico más completo y capaz, dotado de una inteligencia privilegiada de su época. Describió el nervio óptico y el quiasma, estudió el sistema circulatorio de manera muy precisa; describió las valvas de la válvula aórtica y explicó cómo se abrían durante la sístole y cerraban durante la diástole, impidiendo así que la sangre regresara al ventrículo. Definió que el objeto material de la medicina es el cuerpo humano y estableció el límite entre salud y enfermedad. Su obra Canon de la medicina es todo un compendio de los conocimientos existentes a la sazón en este terreno y durante cinco siglos fue el libro de consulta de los médicos, tanto en Oriente como en Occidente (Pérez, 2018).

M. T. Iovchuk (Iovchuk, Oizerman, \& Schipanov, 1978) refiere que Avicena sintió extraordinaria pasión por la filosofía, al punto de ser llamado en su época el segundo maestro, tras Aristóteles que era entonces la autoridad indiscutible. Leyó y comentó los tratados de los filósofos griegos Platón y de Sócrates, maestro de este, así como de otros reconocidos de la Grecia antigua como Heráclito de Éfeso, Anaxágoras, Tales de Mileto y el geómetra Euclides. Define la filosofía como ciencia del ser en cuanto tal. Su objeto no es estudiar tales o cuales manifestaciones del ser, sino el ser en su totalidad. Reconocía la existencia objetiva de la naturaleza. En sus obras científico-naturales se ciñe a los hechos, al experimento y con frecuencia abandona el idealismo y la religión para adoptar una óptica materialista.

Averroes fue filósofo, médico, astrónomo, juez, escritor y profesor, además de elaborar una enciclopedia médica, escribió comentarios sobre la obra de Aristóteles; de ahí que fue conocido como «el Comentador». En su obra Refutación de la refutación (Tahafut altahafut) defiende la filosofía aristotélica frente a las afirmaciones de Al-Ghazali de que la filosofía estaría en contradicción con la religión y sería, por lo tanto una afrenta a las enseñanzas del islam. El parte de la distinción aristotélica entre dos intelectos, el nous pathetikós (intelecto receptivo) y el nous poietikós (intelecto agente), que permitió desligar la reflexión filosófica de las especulaciones míticas y políticas. Se esforzó en aclarar cómo piensa el ser humano y cómo es posible la formulación de verdades 
universales y eternas por parte de seres perecederos. Se distancia de Aristóteles al subrayar la función sensorial de los nervios y al reconocer en el cerebro la localización de algunas facultades intelectivas (imaginación, memoria...). Sitúa el origen de la intelección en la percepción sensible de los objetos individuales y concreta su fin en la universalización, que no existe fuera del alma (el principio de los animales): el proceso consiste en sentir, imaginar y, finalmente, captar el universal. En su obra Tahâfut expone la necesidad de que la ciencia se adecue a la realidad concreta y particular, pues no puede existir conocimiento directo de los universales.

El eje de la filosofía de Averroes es la diferenciación entre el conocimiento humano y el divino. El conocimiento humano, basado en las cosas sensibles, es de los sentidos y de la imaginación; no es un conocimiento objetivo, el cual se define como "unidad e identidad perfecta bajo todo aspecto entre el sujeto y el objeto". El conocimiento humano mantiene necesariamente una inevitable pluralidad al no estar nunca los inteligibles totalmente desligados de las formas imaginativas. Además es incompleto, porque no capta la esencia de las cosas, sino sólo los "accidentes" de las sustancias, (Ruiza, Fernández, \& Tamaro, 2004).

Mahimónides es considerado unánimemente la figura más prominente del judaísmo durante el período medieval. Su riquísima formación intelectual (teológica, filosófica y médica) unida a una profunda espiritualidad dio forma a la figura de un médico altamente humanitario, racional y abnegadamente dedicado a su trabajo. Su legado incluye importantes aportes en el área de la Infectología, su obra refleja un profundo conocimiento de los escritos de médicos griegos e islámicos, destacando Hipócrates y Galeno entre los primeros y Al-Razí (Rhazes) e IbnZuhr (Avenzoar) entre los segundos. Su legado médico consta de diez tratados, entre los cuales destaca la obra 'Aforismos Médicos de Moisés', un conjunto de 1.500 aforismos organizados en 25 capítulos, cada uno versando sobre un área diferente de la medicina. Sus obras -afirma J. Cerda (Cerda, 2009)- de carácter teológico y la célebre 'Guía de Perplejos' alzaron su figura como teólogo y filósofo moderno, siendo esta última considerada la más universal de sus creaciones. Profundo conocedor de la obra de Aristóteles, Maimónides marca la expresión cumbre del racionalismo judío medieval; tuvo confianza plena en la razón, no como recurso infalible de análisis, pero sí como el único instrumento del cual se dispone para la búsqueda de la verdad de Dios y de las cosas (Ruiza, Fernández, \& Tamaro, 2004)

Por su parte en Europa Occidental, a la caída del Imperio Romano, el poderío económico y por tanto ideológico alcanzado por el cristianismo durante el período feudal, trajo como consecuencia la supeditación a la fe religiosa de todas las formas de la producción espiritual. Se impuso la visión determinista y teocentrista de la enfermedad, así como la curación del enfermo únicamente por intermedio de Dios. La tradición médica griega fue estrangulada, al ser eliminada la lectura de sus textos por la Iglesia Católica. A partir de entonces la enseñanza y el estudio de la medicina se realizaban únicamente en los monasterios, decayeron los conocimientos teóricos y no progresaron los que ya existían sobre anatomía y fisiología. La disección de cadáveres fue prohibida durante siglos y 
como consecuencia, persistieron muchos conceptos erróneos de la antigüedad que atrasaron el avance de la medicina.

El estancamiento de los conocimientos médicos en la Edad Media favoreció que la doctrina dogmática de Galeno predominara más allá del medioevo y sirviera de fundamento a la enseñanza de la medicina hasta bien entrado el siglo XVIII.

Posteriormente Descartes, y John Locke fueron médicos cuyo pensamiento ocupan un lugar cimero entre los filósofos de su tiempo. El rasgo fundamental de la filosofía cartesiana es la dualidad cuerpo-alma en el ser humano, coloca la razón en la parte anímica y sostiene al mismo tiempo que los procesos corporales en hombres y animales, al igual que la evolución de la vida en el planeta Tierra, se encuentran regidos por las leyes del movimiento mecánico, -no alcanzó a distinguir la diversa complejidad de las formas del movimiento de los sistemas materiales, ni como las más simples se subordinan a las leyes que rigen otras formas más complejas del movimiento, en la medida que se hacen más complicados los sistemas materiales-.

El alma siempre se había entendido como un principio vital, pero Descartes con su genialidad distintiva la identificará con la mente, sustancia pensante. La vida forma parte entonces de la sustancia extensa que es el cuerpo y se entenderá como producto de mecanismos específicos que operan en él (Roca, 2009). A partir de estos criterios se produjeron toda una serie de estudios basados en la comprensión del cuerpo y de la enfermedad como mecanismos. En sicología, en teoría del conocimiento y en la doctrina del ser fue idealista, no porque considerase que el pensamiento engendraba el ser, sino por creer que la existencia del pensamiento es más indudable y cierta que la existencia del cuerpo, o materia, reconoce la existencia de la sustancia material y la espiritual pero entre ellas se encuentra Dios, como principio supremo. Consideraba al pensamiento, como el único elemento verdadero para llegar a la realidad: cogito ergo sum, pienso luego existo, por tanto, el pensamiento queda validado como método indudable para alcanzar la verdad.

J. Locke fue un filósofo y médico inglés, considerado como uno de los más influyentes pensadores del empirismo inglés y conocido como el «Padre del Liberalismo Clásico». Ilustre exponente del materialismo, fue el fundador del sensualismo materialista según el cual, todo el conocimiento proviene de la percepción sensorial del mundo exterior. La experiencia es la única fuente de las ideas, es el único medio para obtener conocimientos, concibe la mente como una "hoja en blanco", negando que existieran ideas innatas en ella, como decía Descartes, y recalcando que todas las ideas pertenecen, o se adquieren por medio de la experiencia personal.

La teoría de la mente de Locke es frecuentemente citada como el origen de las concepciones modernas de la identidad y del yo, que figuran prominentemente en las obras de filósofos posteriores como Hume, Rousseau y Kant. Locke fue el primero en definir el yo como una continuidad de la conciencia. Postuló que, al nacer, la mente era una pizarra o tabula rasa en blanco. Al contrario de la cartesiana - basada en conceptos preexistentes-, sostuvo que nacemos sin ideas innatas, y que, en cambio, el 
conocimiento solamente se determina por la experiencia derivada de la percepción sensorial. En su obra Ensayo sobre el entendimiento humano (1690), Locke ataca por segunda vez el modo de pensar de su tiempo. Aquí se propuso buscar el origen, la certeza y la extensión del conocimiento humano. Su intención era aplicar el método científico de su tiempo al estudio de las operaciones mentales. A pesar de que Bacon no había profundizado suficientemente en la relación entre las ideas y la experiencia, Locke se preocupa por ello desarrollando el empirismo baconiano, razón por la cual muchos autores lo consideran uno de los más valiosos precursores de la psicología empírica (Uzgalis, 2020).

K. Jasper, filósofo y psiquiatra alemán influyó enormemente tanto en la medicina como en la filosofía. Embebido del pensamiento de Kierkegaard y Nietzsche, se convirtió en uno de los creadores del existencialismo, el cual constituyó un modelo de pensamiento que se centraba en el estudio y reflexión de la condición humana, en la libertad de las personas y en sus responsabilidades como individuos; así como en las emociones y el sentido de la vida, de modo que las reflexiones sobre la libertad personal se convierten en parte central de sus trabajos filosóficos. Al mismo tiempo decide cambiar la perspectiva de la investigación de las enfermedades mentales, y su visión al respecto se convirtió en un clásico de referencia dentro de la bibliografía siquiátrica y cuyas pautas de diagnóstico han servido de inspiración para los procedimientos de diagnósticos modernos (Rovira, 2021).

La figura de Sir William Osler marca el comienzo de importantes conocimientos y aportes en la clínica médica y revoluciona la enseñanza de la medicina. Conocido desde entonces como el padre de la medicina moderna. Su legado fue la enseñanza al lado del paciente; el nuevo currículo, la educación médica y la promoción de la investigación en los estudiantes; el conocimiento de la historia y el humanismo y el amor a la medicina interna. Marcó el comienzo de importantes conocimientos y aportes en la Clínica Médica y revoluciona la enseñanza de la medicina. Conocido desde entonces como el padre de la medicina moderna (Serra, 2015), introdujo la enseñanza de la medicina al lado del enfermo en las salas hospitalarias y la importancia de los conocimientos de Semiología y Técnicas de exploración, así como corroborar los diagnósticos o los errores mediante la autopsia. Innovación sin precedentes en su época. De esta forma hizo aportes importantes en la enseñanza de lo que llamaba "el diagnóstico en la práctica clínica", que por sus características tuvo muchos puntos de encuentro con el denominado "método clínico" actual.

Para Osler la educación en la medicina era el reconocer los aciertos y a la vez las equivocaciones de las que había que aprender y lamentar. "La ciencia y el humanismo no pueden evitar las desgracias implantadas por el error; pero estos dan adquisiciones de conocimientos" (Serra, 2015). Enseñó partiendo del principio de la diversidad biológica, que las enfermedades no eran igual en cada paciente en cuanto a la presentación de todas las manifestaciones clínicas y en el curso clínico de la misma; que los desafíos de la Medicina estaban en que dos cuerpos no eran iguales, no se comportaban enfermos iguales. Consideró la investigación fundamental, sobre todo para la prevención de las 
enfermedades infecciosas. Un gran número de enfermedades infecciosas estudiadas y descritas por él, se presentaban con artralgia y verdaderas artritis, lo que, desde el punto de vista sintomático, constituyen diagnósticos diferenciales para la Reumatología. Tiene más de 1500 publicaciones y aportó a muchas especialidades clínicas, entre las que se encuentra la reumatología.

Fue capaz de transmitirle a sus discípulos la necesidad de conocer y ayudar a los pacientes en sus problemas sociales, la mejor asistencia al pobre que no podía pagar, el principio del alivio del dolor y del sufrimiento humano; pensaba que el profesionalismo médico sería reconocido al practicar siempre la medicina con calidad. Su ética y humanismo lo caracterizó siempre, así como la sencillez, comunicaba conocimientos y experiencias a sus discípulos sin arrogancia ni egoísmo.

Todos estos médicos ilustres, aunque en diversas épocas, desarrollaron sus investigaciones en los más disímiles campos de las ciencias médicas, al mismo tiempo, tuvieron en común, una concepción del mundo, de la vida y la propia humanidad, que los impulsaba hacia la búsqueda y profundización incesante de nuevos conocimientos desde su esfera del saber.

\section{La relación Filosofía-Medicina en la contemporaneidad.}

El desarrollo científico tecnológico hasta nuestros días constituye el resultado lógico del proceso de humanización de la naturaleza por parte del hombre, que ha trascurrido a partir de las soluciones dadas a las necesidades sociales, con un nivel determinado de desarrollo de las condiciones de producción material y espiritual.

En ese proceso de humanización de la naturaleza, el hombre se ha transformado a sí mismo, y hoy no se puede desentender de esa realidad, es por ello que resulta impensable formar científicos, y en especial en las ciencias de la salud, que no sean portadores de un sentido de la vida, de una responsabilidad ante los resultados de su trabajo, sin una ética profesional que los caracterice, sustentada en una profunda concepción del mundo y de lo humano que prestigie la profesión y al mismo tiempo se erija en importante vía para impedir el ejercicio de una medicina deshumanizada, fenómeno que pugna peligrosamente por enraizarse en la práctica médica, en la misma medida que la mercantilización y la medicalización se unen en fuerte abrazo para desplazar al humanismo característico de esta noble profesión.

La medicina -refiere Miguel Kottow- siempre se ha puesto por tarea cuidar y curar a las personas en la situación contingente que es la enfermedad, observación que no es trivial por cuanto indica a la práctica médica como la única que incontestadamente está autorizada para interferir en los procesos naturales, imponiéndole a la enfermedad el artificio de la terapia. Lo cual, antes de ser un problema para la bioética, que por cierto lo será, significa uno frente a la filosofía, porque la vida humana ya no es una realidad con cotos dados, sino un proceso de influencias artificiales que se desarrolla de acuerdo a una intención, un deseo o un interés. (Kottow, 2009) 
Al seguir esta lógica de razonamiento Kottow asegura: que "la medicina contemporánea no puede enfrentar sus problemas sin una reflexión extratemática, porque su ámbito de acción actual y potencial rebasa las tradicionales tareas de curar, paliar y prevenir enfermedades. Al mismo tiempo considera que la filosofía, hace tiempo que abandonó sus inquietudes metafísicas clásicas, se refugió en el cultivo del pensamiento analítico y comenzó a desarrollar una reflexión en torno a la biología, al cuerpo, a la sexualidad y al género, permitiendo que surjan nuevas perspectivas de análisis ante las complejas temáticas que plantean hoy las ciencias de la vida y la tecnociencia en general. La complejidad de estas materias estriba en que ya no se trata de observar e interpretar fenómenos biológicos y estrategias terapéuticas, sino de aprehender que estas realidades están siendo profunda e irreversiblemente transformadas por el ser humano.

El vertiginoso desarrollo científico tecnológico conduce -entre otras problemáticas- a reconsiderar con mayor fuerza la supuesta dicotomía medicina-filosofía, con la que ha venido operando hasta ahora una buena parte de los especialistas.

Miguel Kottow expresaba que en la actualidad ya no tiene sentido seguir reforzando la imagen de aislamiento, separación, puesto que cada vez es más evidente e irrefutable la existencia de profundos nexos "... por cuanto los conocimientos y las competencias biomédicas están llegando a un punto en que la clásica distinción entre natura y cultura desaparece, perdiéndose el ordenamiento ontológico entre lo trascendente, lo que es producto del ser humano, y lo que es naturaleza dada" (Kottow, 2009).

En un primer acercamiento a estas esferas del saber: Filosofía y Medicina, con objetos de estudio bien delimitados y diversos entre sí, puede que solo se capten las diferencias y resulte difícil establecer puntos de contacto, no obstante, al meditar detenidamente se puede establecer una relación: ambas tienen como centro al hombre, la medicina lo estudia desde el ángulo del proceso salud-enfermedad, la filosofía desde su perspectiva generalizadora valora la relación hombre-mundo, de ahí que a la medicina le será muy difícil el abordaje teórico de los problemas que estudia y la comprensión cabal del complejo proceso salud-enfermedad, si no lo hace a partir del conocimiento de la esencia humana y el funcionamiento social del hombre.

Las preguntas iniciales presentes en el pensamiento filosófico, es decir, ¿Qué es el hombre?, ¿Qué es la sociedad?, ¿De dónde venimos?, ¿Cuál es el lugar del hombre en el mundo?, ¿Hacia dónde vamos?, resultan imprescindibles para responder adecuadamente a las cuestiones ontológicas del pensamiento médico de todos los tiempos, tales como: ¿qué es la enfermedad? ¿Qué es la salud?, ¿Cómo interactúan en la vida del hombre?

Tampoco las cuestiones éticas, propias de la práctica médica pueden enfrentarse, si no es a partir de las posiciones axiológicas de un sistema filosófico y el sistema de valores de una sociedad concreta.

A partir del siglo XIX, la medicina se tornó cada vez más invasiva, sin embargo, lo característico y nuevo de la medicina contemporánea, señala Newmn es que no solo interfiere y controla, sino que transforma y reemplaza a la naturaleza al punto que el 
artificio suple y supera lo natural. El espíritu terapéutico que subyace a esta evolución parecería neutralizar toda crítica a la invasión instrumental de la naturaleza, pero por otro lado erige la incógnita acaso habrá un límite ético a la artificialización de lo natural. (Newman, 2007)

¿Qué significa hoy la existencia del sujeto en un mundo dominado por la tecnología?, ¿cuál es el significado de su existencia en la época de la robótica, de la cibernética, de la informática, de la realidad virtual y la inteligencia artificial?

Una de las preocupaciones más importantes de nuestro tiempo tiene que ver con los efectos negativos de diversos factores sobre el ethos médico; entre ellos, quizás los más importantes sean el avance biotecnológico, la mercantilización de la salud, la fragmentación de la atención médica y su creciente deshumanización.

En un mundo globalizado en el que la automatización y la computarización reemplazan a ritmo acelerado el trabajo manual humano y parece apuntar hacia un futuro "posbiológico" y "sobrenatural", aflora la indispensable introspección que debe acompañar la práctica científica en cualquier contexto, la cual entraña un fuerte contenido ético.

Cada vez las ciencias están más cerca del momento en que prácticamente todas las funciones humanas esenciales, físicas y mentales, tendrán su equivalente artificial, ya es una realidad el robot inteligente, el cual representa el prototipo de la unión de los resultados del desarrollo cultural, es una máquina que puede pensar y resolver tareas como un ser humano, aunque física y moralmente no lo sea.

Si a ello le sumamos las posibilidades que representan la clonación de humanos, los avances en el campo de la ingeniería genética, en la biotecnociencia, las nanociencias, las neurociencias, la inteligencia artificial, entre otras, no resulta tan lejano los "replicantes" al estilo de las películas futuristas que inundan la vida cotidiana. Y este escenario tendrá consecuencias positivas indudablemente, pero también implicará riesgos muy negativos para el género humano, y para todo lo vivo en el planeta, si se sigue manteniendo actitudes indiferentes y tolerantes ante las mismas, ¿es posible contemplar impasiblemente como la industria armamentista de los países desarrollados construyen entes autónomos dotados de inteligencia artificial destinados a la guerra?

Infortunadamente el desarrollo científico-tecnológico generado desde los centros de poder hegemónicos actuales, más bien utilizan el conocimiento para dominar la naturaleza con la intención de satisfacer el afán de lucro y no con el propósito de solucionar las necesidades siempre crecientes de la humanidad; tampoco los progresos en la esfera médica están prioritariamente dirigidos hacia la erradicación de las principales causas de muerte de la población mundial. Se enfatiza la investigación de las enfermedades más comunes en países desarrollados y se ignora la agravante ocurrencia de problemas de salud en millares de personas pertenecientes a los países más empobrecidos. 
El quehacer médico siempre ha reconocido que los cánones entre los que es posible actuar, están restringidos por los propios límites naturales de los individuos, por ello admite que mayoritariamente los esfuerzos terapéuticos han sido más paliativos que curativos. Hoy la situación es bien diferente, sobre todo cuando se observa como los avances científicos de las diversas especialidades médicas han llegado a intervenir en las situaciones extremas de la vida humana, con lo cual dejan de ser limítrofes.

Cuando se repara en las infinitas posibilidades que puede brindar la biotecnociencia, los nanofármacos, la nanomedicina, la electroneurobiología, entre otras, apuntan hacia la modificación, la transformación, el cambio y por tanto los límites de la vida humana y el abanico de sus situaciones existenciales, - indica Kottow (2009)- quedan de este modo, cuestionados por la medicina: ¿Qué posibilidades de sobrevivencia tiene un individuo que nace con 500 gramos o menos?, ¿Qué posibilidades de sobrevivencia tiene un individuo que forma parte de un desastre aéreo?

En condiciones naturales, en ambos casos, de lograrse la sobrevivencia, se tendrían serias probabilidades de ingresar al mundo con discapacidades y limitaciones. A ello hay que agregar que la prolongación artificial de la vida a cualquier costo, constituye un relativo fracaso cuando las personas ven su existencia sometida a circunstancias bajo las cuales no desean vivir.

Y esta conciencia de limitaciones afirma Kottow se expresa a través de un lenguaje bioético que llama la atención sobre situaciones como la obstinación terapéutica, las medidas extraordinarias o desproporcionadas, la mantención instrumental de vida, los esfuerzos terapéuticos con severos desmedros de la calidad de vida. Por otro lado, la muerte ha dejado de ser un fenómeno natural de extinción, para volverse un proceso mixto de deterioro y mantención instrumental, posibilidades que han transformado las concepciones acerca del fin de la vida, y han agregado nuevos elementos que suscitan la discusión en torno a tan importante problema para el hombre, como puede ser la definición de muerte.

En la contemporaneidad la revolución científica-tecnológica ha permitido que la práctica médica sea capaz de redelinear las circunstancias esenciales y las situaciones contingentes de la vida humana. Esto ha provocado el surgimiento de múltiples cuestionamientos con relación al quehacer médico, sobre cuando este se enfoca únicamente en alcanzar cuantiosas ganancias y no en función del mejoramiento de la salud de las amplias mayorías.

La realidad que se enfrenta en la actualidad no se puede abordar con una perspectiva conservadora de los procesos de salud y la descripción formalista de los problemas médicos y sus relaciones con otras esferas de la sociedad en general.

\section{Conclusiones}

- Ignorar los vínculos Filosofía-Medicina, expresa la incapacidad para comprender los profundos cambios que se están operando en el conocimiento humano, en los 
modos de concebirlo, producirlo y socializarlo, así como la tendencia hacia la integración.

- Resulta inadmisible la comprensión de una esfera médica absolutamente desvinculada de las múltiples relaciones sociales con las cuales interactúa, al mismo tiempo impide una plena comprensión de su papel como parte integrante del engranaje social, y se presenta como un organismo aislado, como un ente autónomo y despojado de los indisolubles lazos que la unen a la sociedad.

- Formar científicos y en especial en ciencias médicas significa que además de los conocimientos concretos de su especialidad, debe ser portador de una posición ante la vida, de una responsabilidad ante los resultados de su trabajo sustentados en una profunda concepción del mundo y de lo humano que honre la profesión.

\section{Referencias Bibliográficas}

Campohermoso Rodríguez O, S. S. (2017). org. Recuperado el 2020, de http://www.revistasbolivianas.org.bo/pdf/chc/v55n1/v55n1_a08.pdf.

Cerda, J. (2009). Mahimónides. Recuperado el 29 de Mayo de 2021, de Scielo: https://scielo.conicyt.cl/scielo.php?pid=S071610182009000500013\&script=sci_ arttext\&tlng=pt

Echarri, H. J. (2010). Relaciones entre las ciencias sociales y el campo de la salud. Revista de Psicología de Universiddad de Antioquía versión on line ISSN 2145-4892, 2(2).

Iovchuk, M., Oizerman, T., \& Schipanov, E. (1978). Historia de la Filosofía. Moscú: Progreso.

Kottow Miguel. (s.f.). La simbiosis entre medicina y filosofía. Obtenido de http://www.saludpublica.uchile.cl/academicos/bioetica/103061/miguel-kottowlang.

Llanes, B. R. (2012). Recuperado el 2018, de Infomed Universidad Virtual Fajardo Educación Médica en Red.

Mainetti, J. A. (marzo de 2006). La medicalización de la vida. Recuperado el 2017, de Electroneurobiología: http://electroneubio.secyt.gov.ar/index2.htm>

Mainetti, J. (s.f.). La crisis de la razón médica. Introducción a la filosofía de la medicina. Obtenido de : htp:// electroneubio.secyt.gov.art/index2.htm

Newman, V. B. (2007). The artificial and the natural. Cambridge/London: MIT Press.

O’Connor, A. J. (2005). Medicina y Filosofía. Hospital Central, 2-3.

Peña, A. (2004). Medicina y Filosofía: abordaje filosófico de algunos problemas de la medicina actual. Anales de la Facultad de Medicina, 65(1), 65-72. 
Pérez, A. J. (Marzo de 2018). Scielo. Recuperado el 29 de Mayo de 2021, de https://scielo.conicyt.cl/scielo.php?pid=S071610182009000500013\&script=sci_ arttext\&tlng=pt

Roca Jusmeta, L. (15 de mayo de 2009). Filosofía y Medicina. Revista Médica de Homeopatía.

Rodríguez, A., \& P, J. (2012). Relación entre medicina y filosofía. Revista de la Sociedad Boliviana de Pediatrñia, 51(1).

Rovira, S. I. (2021). Psicología y mente. Recuperado el 29 de Mayo de 2021, de https://psicologiaymente.com/biografias/karl-jaspers

Ruiza, M., Fernández, T., \& Tamaro, E. (2004). Biografía de Averroes. Recuperado el 3 de Junio de 2021, de Biografías y Vidas: https://www.biografías y vidas.com

Ruiza, M., Fernández, T., \& Tamaro, E. (2004). Biografía de Galeno. Recuperado el 3 de Junio de 2021, de Biografías y vidas: https://www.biografiasyvidas.com/biografia/g/galeno.htm

Ruiza, M., Fernández, T., \& Tamaro, E. (2004). Biografía de Maimonides. Recuperado el 31 de mayo de 2021, de Biografías y vidas: https://www.biografiasyvidas.com/biografia/m/maimonides.htm

Serra, V. M. (2015). Recuperado el 28 de Mayo de 2021, de Scielo: http://scielo.sld.cu/scielo.php?script=sci_arttext\&pid=S18175996201500030001 $4 \& \operatorname{lng}=$ es.

Sigerist A. (2008). On the sociology of medicine. En: Javier Mariategui Chiappe. La Medicina como ciencia social. . Revista Neurosiquiatría , 71.

Taborda Alzate Jhon M., O. J. (2008). La medicina como filosofía. CES MED, 22(2), 127-31.

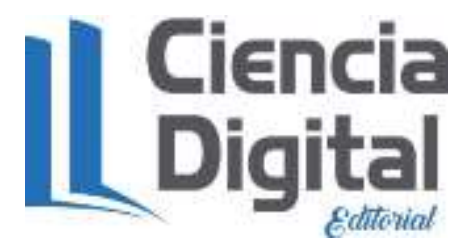




\section{PARA CITAR EL ARTÍCULO INDEXADO.}

Fernández Valderrama, C. J., Rodríguez Álvarez, B., Fernández Garrido, Z., \& Velastegui López, E. (2021). ¿El hacer médico necesita del pensar filosófico?. AlfaPublicaciones, 3(4), 39-54. https://doi.org/10.33262/ap.v3i4.110

\section{Ciencia}

El artículo que se publica es de exclusiva responsabilidad de los autores y no necesariamente reflejan el pensamiento de la Revista Alfa Publicaciones.

El artículo queda en propiedad de la revista y, por tanto, su publicación parcial y/o total en otro medio tiene que ser autorizado por el director de la Revista Alfa Publicaciones.
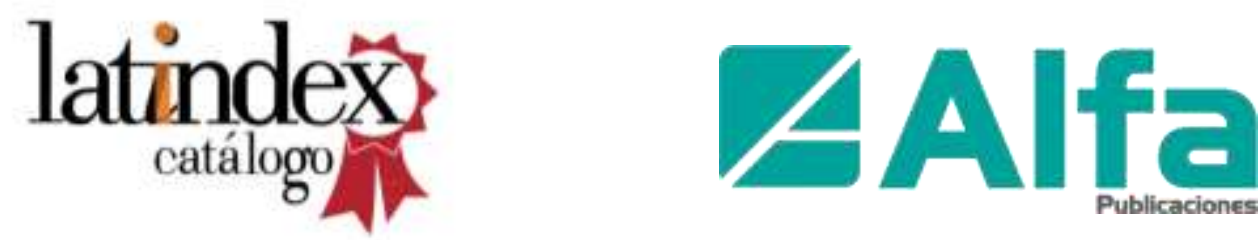